\title{
Cardiovascular risk factors and cognitive decline in older people with type 2 diabetes
}

\author{
Insa Feinkohl ${ }^{1}$ • Markéta Keller ${ }^{1}$ - Christine M. Robertson ${ }^{1}$ • Joanne R. Morling ${ }^{1}$ • \\ Stela McLachlan ${ }^{1}$ - Brian M. Frier ${ }^{2,3}$ • Ian J. Deary ${ }^{3,4}$ • Mark W. J. Strachan ${ }^{5}$ • \\ Jackie F. Price ${ }^{1}$
}

Received: 12 December 2014 / Accepted: 16 March 2015 /Published online: 7 April 2015

(C) The Author(s) 2015. This article is published with open access at Springerlink.com

\begin{abstract}
Aims/hypothesis The aim of this work was to assess the role of well-established cardiovascular risk factors in the late-life cognitive decline of patients with type 2 diabetes.

Methods Data from 831 participants (aged 60-75 years) attending the 4 year follow-up of the Edinburgh Type 2 Diabetes Study (ET2DS) were used. Smoking history (pack-years), BP, $\mathrm{HbA}_{1 \mathrm{c}}$, plasma glucose and cholesterol were determined at baseline clinics (single time measurements) and/or from serial data recorded on a clinical management database from diagnosis until recruitment ('historical' data). Principal component analysis derived a factor, $g$, of general ability from seven cognitive tests. Linear regression models of follow-up $g$ were adjusted for baseline $g$ to represent 4 year cognitive change. 'Accelerated late-life cognitive decline' was defined as scor-
\end{abstract}

Electronic supplementary material The online version of this article (doi:10.1007/s00125-015-3581-0) contains peer-reviewed but unedited supplementary material, which is available to authorised users.

Insa Feinkohl

ifeinkohl@gmail.com

1 Centre for Population Health Sciences, Medical School, University of Edinburgh, Teviot Place, Edinburgh EH8 9AG, Scotland, UK

2 The Queen's Medical Research Institute, University of Edinburgh, Edinburgh, Scotland, UK

3 Centre for Cognitive Ageing and Cognitive Epidemiology, University of Edinburgh, Edinburgh, Scotland, UK

4 Psychology in the School of Philosophy, Psychology and Language Sciences, University of Edinburgh, Edinburgh, Scotland, UK

5 Metabolic Unit, Western General Hospital, Edinburgh, Scotland, UK ing in the lowest tertile of ' 4 year cognitive change' regression scores. Analyses controlled for age and sex.

Results A baseline history of moderate/heavy smoking ( $\geq 10$ pack-years) and a $1 \%$ increased historical $\mathrm{HbA}_{1 \mathrm{c}}$ (equivalent to an increase by $11 \mathrm{mmol} / \mathrm{mol}$ ) predicted a $64 \%$ (OR 1.64; $95 \%$ CI $1.14,2.34 ; p=0.007)$ and $21 \%$ (OR $1.21 ; 95 \%$ CI $1.00,1.45 ; p=0.046)$ increased risk of accelerated cognitive decline, respectively. When treated as continuous measures, higher pack-years, historical $\mathrm{HbA}_{1 \mathrm{c}}$ and historical $\mathrm{BP}$ emerged as significant independent predictors of 4 year decline in $g$ (standardised $\beta$ range -0.07 to -0.14 ; all $p \leq 0.05$ ). Conclusions/interpretation Increased smoking and poorer glycaemic control (with relatively weaker findings for BP) during the life-course were independently associated with accelerated late-life cognitive decline. Where possible, evaluation is warranted of these risk factors as targets for intervention to reduce the burden of cognitive impairment in diabetes.

Keywords Blood glucose $\cdot$ Blood pressure $\cdot$ Cardiovascular risk · Cholesterol - Cognitive impairment - Glycaemic control $\cdot$ Hyperglycaemia $\cdot$ Older age $\cdot$ Smoking $\cdot$ Type 2 diabetes

$\begin{array}{ll}\begin{array}{l}\text { Abbreviations } \\ \text { ACCORD-MIND }\end{array} & \begin{array}{l}\text { Action to Control Cardiovascular } \\ \text { Risk in Diabetes } \\ \text { Atherosclerosis Risk in Communities }\end{array} \\ \text { ARIC } & \text { Borkowski Verbal Fluency test } \\ \text { BVFT } & \text { Digit Symbol Coding } \\ \text { DSC } & \text { Edinburgh Type 2 Diabetes Study } \\ \text { ET2DS } & \text { Factor of general cognitive ability } \\ g & \text { Logical Memory } \\ \text { LM } & \text { Letter-Number Sequencing } \\ \text { LNS } & \text { Matrix Reasoning }\end{array}$


TMT-B

WAIS-III

Trail-Making Test-B

Wechsler Adult Intelligence Scale, third edition

\section{Introduction}

People with type 2 diabetes are at greater risk of cognitive impairment in later life than their non-diabetic peers [1]. With the prevalence of diabetes expected to reach $15 \%$ in the USA within the current decade [2], researchers have attempted to identify underlying pathophysiological mechanisms and potentially modifiable risk factors that may be responsible for the observation. For instance, based on competition of insulin with $\beta$-amyloid peptide for degradation and recent reports identifying the amyloid precursor amylin, which is cosecreted with insulin from the pancreas, in brains of patients with diabetes (but not in diabetes-free controls), increased concentrations of $\beta$-amyloid and amylin are candidate links between type 2 diabetes and cognitive impairment $[3,4]$. The role of vascular disease has also received increasing attention of late due to an increased prevalence in type 2 diabetes and plausible biological mechanisms linking the two conditions. Amylin, for instance, is known to contribute to cardiovascular disease, so similar effects on the vascular system of the brain may be plausible [4]. Recent epidemiological evidence has also shown a direct association between cognitive decline and markers of symptomatic and asymptomatic vascular disease in people with type 2 diabetes [5]. These observations suggest a potential role for smoking, hypertension, adverse lipid profiles and hyperglycaemia, all of which are established risk factors for adverse cardiovascular outcomes, in the development of cognitive impairment in people with type 2 diabetes. If such a role is repeatedly demonstrated, then future clinical trials targeting these risk factors should consider inclusion of cognitive endpoints (e.g. to determine whether a change in the threshold for existing clinical intervention may be indicated).

In the general population, hypercholesterolaemia, hypertension, smoking and hyperglycaemia have each been associated with a poorer level of cognitive function [6-8]. Lower childhood intelligence, however, potentially leads to increased vascular risk, as well as to lower cognitive ability and greater cognitive decline in later life [9]. Therefore, studies with a prospective design and, preferably, with a measure of premorbid cognitive ability, are required to help determine the direction of relationships between vascular risk factors and cognitive decline. Compared with previous prospective studies in the general population that suggest a degree of complexity and do not always provide a consistent picture of vascular risk and cognitive impairment $[10,11]$, such studies in diabetic populations are much more scarce and often sub-optimal in design not least due to the neglect of consideration of pre- morbid ability [12]. For smoking in particular, this type of evidence was lacking until a recent analysis of the Fremantle Diabetes Study, which showed that midlife smoking predicted an increased risk of future cognitive impairment [13].

Using data from a large prospective cohort of older adults with type 2 diabetes, the Edinburgh Type 2 Diabetes Study (ET2DS), we tested the associations of serum cholesterol, BP, glycaemic control and smoking with late-life cognitive decline. Given known strong correlations among vascular risk factors themselves, it was important to evaluate the relative independence of their contributions in our cohort. Our analyses had the additional advantage of using 'historical' data on $\mathrm{BP}$ and $\mathrm{HbA}_{1 \mathrm{c}}$ covering the time between diabetes diagnosis and attendance at the clinic.

\section{Methods}

\section{Study population}

The baseline clinic of the ET2DS was attended by 1,066 community-dwelling adults aged 60-75 years [14], of whom 831 returned 4 years later. Ethical approval was obtained from the Lothian Medical Research Ethical Committee. Examinations complied with the Declaration of Helsinki and participants gave full written consent. Details of study recruitment and examination have been described previously [15].

\section{Baseline clinical examination and historical data}

Data obtained at baseline ('clinic data') and from a clinical management database between diabetes diagnosis and attendance at the baseline clinic ('historical data') were used. At baseline, blood samples were taken following an overnight fast to measure $\mathrm{HbA}_{1 \mathrm{c}}$, plasma glucose, serum LDLcholesterol and HDL-cholesterol. The ratio of HDL-cholester$\mathrm{ol} /$ total cholesterol was used ('cholesterol') in subsequent analysis as such ratios may be preferable to non-ratio measures in cardiovascular risk prediction [16].

Questionnaires administered at baseline obtained data on education, smoking and medical history. Medical history data, together with Scottish Morbidity Record Data, responses on the World Health Organization chest pain questionnaire and 12-lead ECG data, identified angina, transient ischaemic attack, stroke and myocardial infarction [14]. Hypertension was defined as systolic BP $\geq 140 \mathrm{mmHg}$ and/or diastolic BP $\geq 85 \mathrm{mmHg}$ at the clinic visit and/or when participants self-reported taking medication to lower BP. Hypercholesterolaemia was defined as plasma total cholesterol $\geq 5 \mathrm{mmol} / \mathrm{l}$ and/or when participants self-reported taking medication to lower blood lipids.

Questionnaire items ascertained current and previous smoking, including years since cessation and number of 
cigarettes, cigars or ounces of tobacco smoked per day. Each self-reported cigar smoked was converted to equal four cigarettes; each 20 cigarettes were converted to equal one pack. The number of packs was multiplied by the number of years participants had smoked to obtain 'pack-years' (1 pack-year is equivalent to 20 cigarettes/day for 1 year). For pipe smoking, $7,300 \mathrm{~g}$ of tobacco were equivalent to 1 pack-year. The measure of pack-years was additionally categorised into participants who had never smoked or had a history of light smoking $(<10$ pack-years) and those with a history of moderate to heavy smoking ( $\geq 10$ pack-years).

Historical data from the Lothian Diabetes Register between 1988 and 2007 (the year of study recruitment) were used to obtain time-weighted mean measures of systolic and diastolic BP ('historical systolic BP'; 'historical diastolic BP') and $\mathrm{HbA}_{1 \mathrm{c}}$ ('historical $\mathrm{HbA}_{1 \mathrm{c}}$ '). Data from between one reading and 65 readings (median 19 readings, interquartile range 14 25 readings) were available for each individual and were used to calculate the time-weighted mean. Assessments captured by the register spanned between 0 years (single assessment) and 19.8 years (median 10.4 years, interquartile range $7.3-$ 13.6 years). 'Poor glycaemic control' was defined as a historical $\mathrm{HbA}_{1 \mathrm{c}}>7 \%$ (>53 mmol/mol). 'Poor BP control' was defined as historical systolic $\mathrm{BP} \geq 140 \mathrm{mmHg}$ and/or historical diastolic $\mathrm{BP} \geq 85 \mathrm{mmHg}$. Clinical characteristics of the study population are shown in Table 1 .

\section{Cognitive assessment}

At baseline and at year 4, seven age-sensitive tests of cognitive function were administered. Executive function was measured by the Trail-Making Test-B (TMTB) and the Borkowski Verbal Fluency Test (BVFT). The Digit Symbol Coding (DSC) subtest of the Wechsler Adult Intelligence Scale, third edition (WAIS-III) ascertained processing speed [17]. The Matrix Reasoning (MR) subtest of the WAIS-III measured non-verbal reasoning and the Letter-Number Sequencing (LNS) subtest measured working memory. The Logical Memory (LM) and Faces subtests of the Wechsler Memory Scale, third edition, assessed verbal and nonverbal declarative memory, respectively [18]. Scores on the combined junior and senior synonyms of the Mill Hill Vocabulary Scale (MHVS) [19], which have been shown to correlate with scores on other vocabularybased tests [20], estimated pre-morbid cognitive ability. Vocabulary-based tests are used in this function on the grounds that vocabulary is part of 'crystallised' ability and hence is relatively immune to age-related cognitive decline [21]. The criteria used to identify participants with possible dementia by year 4 have been described previously [5].

\section{Statistical analyses}

Distribution was approximately normal for all measured variables; for TMTB and clinic plasma glucose, normal distribution was achieved following transformation to their natural logarithms. Normal distribution for pack-years was achieved using square root transformation. Missing data on cognitive ability tests other than Mill Hill Vocabulary (0.6$1.7 \%$ at baseline; $0.8-4.7 \%$ at year 4 ) were imputed, as described in detail previously [5]. Since people who perform well on one cognitive test tend to perform well on another, data reduction techniques may be applied to batteries of $\operatorname{cog}$ nitive tests with complete data on all individual tests to obtain a factor $g$ of general ability [22]. The use of $g$ is advantageous, because it is relatively unaffected by measurement error. Here, components with eigenvalues $>1$ were extracted in principal component analysis. All seven cognitive tests (those other than Mill Hill Vocabulary) loaded on a single component, accounting for $44.74 \%$ and $47.44 \%$ of variance at baseline and at year 4 , respectively. Factor loadings ranged between 0.47 (Faces) and 0.80 (TMTB) at baseline and between 0.51 (Faces) and 0.81 (TMTB) at year 4 [5].

Linear regression analyses regressed cognitive function at year 4 and cognitive change between baseline and year 4 on each of the vascular risk factors (see Table 2 for analyses of $g$; see Electronic supplementary material [ESM] Table 1 for analyses of individual cognitive tests and 'estimated lifetime cognitive change', determined by adjustment of year 4 cognitive test scores for Mill-Hill Vocabulary). Four year cognitive change was represented by adjustment of year 4 scores for baseline scores (the procedure to ensure that baseline and year $4 \mathrm{~g}$ were standardised on the same sample has been described previously [5]). This method may be preferable to raw change scores because it is less dependent on individual differences in initial cognitive status [23].

Regression analyses of cognitive function at year 4 were adjusted for age and sex; those of 4 year cognitive change were adjusted for baseline cognitive scores, age and sex, before myocardial infarction, angina, transient ischaemic attack, stroke ('vascular disease') and duration of diabetes, and MillHill Vocabulary were controlled for in two separate steps. For $g$, analyses that survived this adjustment were additionally controlled for mode of glucose-lowering treatment. Finally, pack-years, historical systolic $\mathrm{BP}$, historical $\mathrm{HbA}_{1 \mathrm{c}}$ and cholesterol were selected a priori (with the aim of avoiding multicollinearity) to be entered as predictors in a single model to ascertain the relative independence of associations. Additional categorisation of cognitive outcomes allowed the calculation of ORs for 'poor' cognitive function (scoring in lowest vs highest tertile of follow-up $g$ ) and 'accelerated' 4 year cognitive decline (lowest vs highest tertile of follow-up $g$ adjusted 
Table 1 Baseline demographics and clinical characteristics of attendees of the year 4 follow-up

\begin{tabular}{|c|c|c|c|c|c|c|c|}
\hline \multirow[t]{2}{*}{ Characteristic/demographic } & \multicolumn{2}{|c|}{ Total sample } & \multicolumn{2}{|l|}{ Men } & \multicolumn{2}{|c|}{ Women } & \multirow[t]{2}{*}{$p$ value $^{\mathrm{a}}$} \\
\hline & $N$ & $\begin{array}{l}\text { Mean } \pm \mathrm{SD} \text {, median } \\
\text { (interquartile range) } \\
\text { or } n(\%)\end{array}$ & $N$ & $\begin{array}{l}\text { Mean } \pm \mathrm{SD} \text {, median } \\
\text { (interquartile range) } \\
\text { or } n(\%)\end{array}$ & $N$ & $\begin{array}{l}\text { Mean } \pm \mathrm{SD} \text {, median } \\
\text { (interquartile range) } \\
\text { or } n(\%)\end{array}$ & \\
\hline Age (years) & 831 & $67.7 \pm 4.2$ & 430 & $67.8 \pm 4.1$ & 401 & $67.6 \pm 4.3$ & 0.569 \\
\hline Duration of diabetes (years) & 824 & $6(3-11)$ & 425 & $6(4-11)$ & 399 & $6(3-10)$ & 0.236 \\
\hline Insulin \pm oral glucose-lowering treatment & 830 & $139(16.7)$ & 429 & $66(15.4)$ & 399 & $73(18.2)$ & \multirow[t]{3}{*}{0.756} \\
\hline Oral glucose-lowering treatment alone & 830 & $526(63.4)$ & 429 & $276(64.3)$ & 399 & $250(62.4)$ & \\
\hline Diet alone as glucose-lowering treatment & 830 & $165(19.9)$ & 429 & $87(20.3)$ & 399 & $78(19.5)$ & \\
\hline Lipid-lowering treatment & 831 & $719(86.5)$ & 430 & $368(85.6)$ & 401 & $351(87.5)$ & 0.411 \\
\hline Hypercholesterolaemia & 831 & $770(92.7)$ & 430 & 394 (91.6) & 401 & $376(93.8)$ & 0.238 \\
\hline Total cholesterol (mmol/l) & 826 & $4.3 \pm 0.9$ & 427 & $4.2 \pm 0.8$ & 399 & $4.5 \pm 0.9$ & $<0.001$ \\
\hline HDL/total cholesterol & 826 & $0.30 \pm 0.09$ & 427 & $0.29 \pm 0.08$ & 399 & $0.32 \pm 0.08$ & $<0.001$ \\
\hline Historical systolic BP (mmHg) & 825 & $139 \pm 11$ & 425 & $139 \pm 10$ & 400 & $139 \pm 11$ & 0.509 \\
\hline Historical diastolic BP (mmHg) & 825 & $79 \pm 6$ & 425 & $79 \pm 6$ & 400 & $79 \pm 6$ & 0.189 \\
\hline Clinic systolic BP (mmHg) & 829 & $133 \pm 16$ & 430 & $133 \pm 15$ & 399 & $132 \pm 17$ & 0.575 \\
\hline Clinic diastolic BP (mmHg) & 829 & $69 \pm 9$ & 430 & $70 \pm 9$ & 399 & $67 \pm 8.7$ & $<0.001$ \\
\hline Antihypertensive treatment & 826 & $684(82.8)$ & 426 & $359(84.3)$ & 400 & $325(81.3)$ & 0.250 \\
\hline Hypertension & 831 & $729(87.7)$ & 430 & $384(89.3)$ & 401 & $345(86.0)$ & 0.151 \\
\hline Poor blood pressure control & 825 & $389(47.2)$ & 425 & $193(45.4)$ & 401 & $196(49.0)$ & 0.302 \\
\hline Pack-years & 803 & $6(0-30)$ & 410 & $17(0-40)$ & 393 & $0(0-20)$ & $<0.001$ \\
\hline Never smoked/history of light smoking & 803 & $425(52.9)$ & 420 & $166(40.5)$ & 393 & $259(65.9)$ & \multirow[t]{2}{*}{$<0.001$} \\
\hline History of moderate/heavy smoking & 803 & $378(47.1)$ & 420 & $422(59.5)$ & 393 & $134(34.1)$ & \\
\hline Historical $\mathrm{HbA}_{1 \mathrm{c}}(\%)$ & 825 & $7.4 \pm 0.9$ & 425 & $7.3 \pm 0.9$ & 400 & $7.5 \pm 0.9$ & \multirow[t]{2}{*}{0.061} \\
\hline Historical $\mathrm{HbA}_{1 \mathrm{c}}(\mathrm{mmol} / \mathrm{mol})$ & 825 & $57 \pm 10$ & 425 & $56 \pm 10$ & 400 & $59 \pm 10$ & \\
\hline Clinic $\mathrm{HbA}_{1 \mathrm{c}}(\%)$ & 804 & $7.4 \pm 1.1$ & 415 & $7.4 \pm 1.2$ & 389 & $7.4 \pm 1.1$ & \multirow[t]{2}{*}{0.386} \\
\hline Clinic $\mathrm{HbA}_{1 \mathrm{c}}(\mathrm{mmol} / \mathrm{mol})$ & 804 & $57 \pm 12$ & 415 & $57 \pm 13$ & 389 & $57 \pm 12$ & \\
\hline Clinic plasma glucose (mmol/l) & 821 & $7.2(6.2-8.4)$ & 424 & $7.3(6.3-8.5)$ & 397 & $7.1(6.0-8.2)$ & 0.059 \\
\hline Poor glycaemic control & 825 & $560(67.9)$ & 425 & $279(65.6)$ & 400 & $281(70.3)$ & 0.157 \\
\hline History of severe hypoglycaemia & 816 & $77(9.4)$ & 425 & $33(7.8)$ & 391 & $44(11.3)$ & 0.089 \\
\hline Diabetic retinopathy & 819 & $266(32.5)$ & 424 & $155(36.6)$ & 395 & $111(28.1)$ & 0.010 \\
\hline Waist-hip ratio & 828 & $0.97 \pm 0.08$ & 429 & $1.00 \pm 0.06$ & 399 & $0.93 \pm 0.07$ & $<0.001$ \\
\hline Carotid intima-media thickness (mm) & 775 & $1.00 \pm 0.17$ & 399 & $1.03 \pm 0.18$ & 376 & $0.96 \pm 0.16$ & $<0.001$ \\
\hline Myocardial infarction & 831 & $111(13.4)$ & 430 & $83(19.3)$ & 401 & $28(7.0)$ & $<0.001$ \\
\hline Angina & 831 & $222(26.7)$ & 430 & $137(31.9)$ & 401 & $85(21.2)$ & 0.001 \\
\hline Stroke & 831 & $44(5.3)$ & 430 & $32(7.4)$ & 401 & $12(3.0)$ & 0.004 \\
\hline Transient ischaemic attack & 831 & $27(3.2)$ & 430 & $16(3.7)$ & 401 & $11(2.7)$ & 0.427 \\
\hline
\end{tabular}

Total $N=831$

Poor glycaemic control was defined as historical $\mathrm{HbA}_{1 \mathrm{c}}>7 \%(>53 \mathrm{mmol} / \mathrm{mol})$. Poor blood pressure control was defined as historical systolic $\mathrm{BP}$ $\geq 140 \mathrm{mmHg}$ and/or historical diastolic $\mathrm{BP} \geq 85 \mathrm{mmHg}$. Hypertension was defined as systolic $\mathrm{BP} \geq 140 \mathrm{mmHg}$ and/or diastolic $\mathrm{BP} \geq 85 \mathrm{mmHg}$ at the clinic visit and/or self-reported medication prescribed by a doctor to lower blood pressure. Hypercholesterolaemia was defined as plasma total cholesterol $\geq 5 \mathrm{mmol} / 1$ and/or when a participant self-reported medication prescribed by a doctor to lower blood lipid level. Never smoked/history of light smoking was defined as $<10$ pack-years. History of moderate/heavy smoking was defined as $\geq 10$ pack-years. Carotid intima-media thickness was measured at year 1 follow-up. Diabetic retinopathy was defined as mild or moderate/severe retinopathy on seven-field retinal photographs. History of severe hypoglycaemia was defined as self-reported history of at least one episode of hypoglycaemia requiring assistance by another person (for details, see [32]) ${ }^{a} p$ value for sex difference in $\chi^{2}$ tests or $t$ tests

for baseline $g$ ) using logistic regression. All linear and logistic regression analyses of $g$ were repeated separately with stratification by sex (see Table 3 and ESM Table 2), on the basis of original rather than imputed data, and with exclusion of cases with possible dementia $(n=4)$; none of the findings were altered in these latter two steps unless noted in the text. 
Table 2 Clinical predictors of global cognitive performance $(g)$ at year 4 and of 4 year change in $g$ adjusted for MHVS and other covariates

\begin{tabular}{|c|c|c|c|c|}
\hline \multirow[t]{2}{*}{ Predictor } & \multirow{2}{*}{$\begin{array}{l}\beta \text { adjusted } \\
\text { for age and sex }\end{array}$} & \multicolumn{3}{|c|}{4 year cognitive change } \\
\hline & & $\begin{array}{l}\text { Adjusted for age, } \\
\text { sex, baseline } g\end{array}$ & $\begin{array}{l}\text { Adjusted for age, sex, } \\
\text { baseline } g \text {, covariates }\end{array}$ & $\begin{array}{l}\text { Adjusted for age, sex, baseline } \\
g \text {, covariates, MHVS }\end{array}$ \\
\hline Cholesterol & $0.08(0.017)$ & $0.01(0.823)$ & $0.00(0.938)$ & $-0.01(0.981)$ \\
\hline Hypercholesterolaemia & $0.02(0.524)$ & $0.02(0.630)$ & $0.02(0.543)$ & $0.02(0.601)$ \\
\hline Historical systolic BP & $0.00(0.992)$ & $-0.07(0.052)$ & $-0.07(0.067)$ & $-0.07(0.051)$ \\
\hline Historical diastolic BP & $0.05(0.149)$ & $-0.01(0.750)$ & $-0.02(0.602)$ & $-0.03(0.375)$ \\
\hline Poor BP control & $0.01(0.815)$ & $-0.01(0.810)$ & $0.00(0.931)$ & $-0.01(0.711)$ \\
\hline Hypertension & $0.01(0.740)$ & $0.00(0.968)$ & $0.01(0.764)$ & $0.03(0.470)$ \\
\hline Pack-years & $-0.15(<0.001)$ & $-0.15(<0.001)$ & $-0.14(<0.001)$ & $-0.12(0.002)$ \\
\hline Historical $\mathrm{HbA}_{1 \mathrm{c}}$ & $-0.11(0.001)$ & $-0.11(0.001)$ & $-0.10(0.005)$ & $-0.10(0.004)$ \\
\hline Poor glycaemic control & $-0.03(0.337)$ & $-0.05(0.149)$ & $-0.05(0.144)$ & $-0.04(0.264)$ \\
\hline Clinic plasma glucose & $0.04(0.296)$ & $0.03(0.350)$ & $0.04(0.216)$ & $0.04(0.296)$ \\
\hline
\end{tabular}

Data are shown as standardised $\beta$ coefficients ( $p$ values). $N=778-823$

Results are from multiple linear regression models performed separately for each risk factor. Outcome variable is $g$ at year 4 . Adjustment of year 4 scores for baseline scores represented 4 year change in cognitive performance. Pack-years are square root transformed. Clinic plasma glucose was transformed to its natural logarithm. Hypertension was defined as systolic $\mathrm{BP} \geq 140 \mathrm{mmHg}$ and/or diastolic BP $\geq 85 \mathrm{mmHg}$ and/or self-reported medication prescribed by a doctor to lower BP. Hypercholesterolaemia was defined as plasma total cholesterol $\geq 5 \mathrm{mmol} / \mathrm{l}$ and/or self-reported medication prescribed by a doctor to lower blood lipids level. Poor glycaemic control was defined as historical $\mathrm{HbA}_{1 \mathrm{c}}>7 \%(>53 \mathrm{mmol} / \mathrm{mol})$. Poor BP control was defined as historical systolic $\mathrm{BP} \geq 140 \mathrm{mmHg}$ and/or historical diastolic $\mathrm{BP} \geq 85 \mathrm{mmHg}$. Covariates are baseline myocardial infarction, transient ischaemic attack, stroke, angina, duration of diabetes. MHVS, Mill Hill Vocabulary Scale

\section{Results}

\section{Cohort characteristics}

Eight hundred and thirty-one participants (51.7\% male) of the ET2DS attended for follow-up cognitive testing. Clinical and cognitive differences between attenders and non-attenders have been described previously [5]. Baseline clinical and demographic characteristics of attendees (forming the study population for this analysis) are shown in Table 1. Mean age at baseline was 67.7 years, with median time since diabetes diagnosis of 6 years. Almost all participants suffered from hypercholesterolaemia (92.7\%) and/or hypertension (87.7\%); a majority had poor glycaemic control (67.9\%) and around half $(47.2 \%)$ had poor blood pressure control, according to our pre-specified criteria, respectively.

\section{Vascular risk and cognition in total sample}

Blood pressure Mill Hill Vocabulary was unrelated to historical diastolic $(p=0.076)$ or systolic BP $(p=0.701)$. Higher historical systolic BP was marginally associated with a steeper 4 year decline in $g$ (Table 2), mainly due to an association with reasoning abilities (Matrix Reasoning) (ESM Table 1). Adjusting the association with 4 year decline in $g$ for vascular disease, disease duration and Mill Hill Vocabulary did not alter the results in terms of effect size or statistical significance, and adjusting for glucose-lowering treatment led to statistical significance ( standardised $\beta-0.07 ; p=0.047$ ). Upon exclusion of individuals with possible dementia, the finding for $g$ (standardised $\beta-0.06 ; p=0.069$ ) was attenuated. When analyses were repeated with non-imputed rather than imputed data, the marginal association with 4 year decline in $g$ lost statistical significance $(-0.06 ; p=0.125)$. BP was unrelated to risk of poor cognitive outcome (Table 3 ).

Cholesterol People with higher cholesterol levels tended to have higher Mill Hill Vocabulary $(r=0.07 ; p=0.054)$. A statistically significant association of higher cholesterol level with lower $g$ did not survive adjustment for Mill Hill Vocabulary (ESM Table 1) or for baseline $g$ (Table 2), and logistic regression analyses showed that cholesterol was unrelated to risk of poor cognitive outcome (data not shown).

Smoking Pack-years was unrelated to Mill Hill Vocabulary ( $p=0.157)$, but was associated with lower $g$ at baseline and with accelerated decline in $g$ (Table 2). Neither inclusion of vascular disease, disease duration and Mill Hill Vocabulary (Table 2) nor of glucose-lowering treatment (data not shown) altered the results. In addition to $g$, pack-years was associated with decline on all of the seven individual tests (ESM Table 1). Overall, individuals with a history of moderate to heavy smoking had a $64 \%$ increased odds of accelerated 4 year cognitive decline after controlling for age and sex. To illustrate, independent of age and sex, each additional pack-year was associated with $1 \%$ increased odds of accelerated 4 year decline (Table 3). 
Table 3 Incremental odds of poor cognitive performance at year 4 and of accelerated 4 year cognitive decline according to preceding vascular risk factors

\begin{tabular}{|c|c|c|c|c|}
\hline Risk factor & Poor cognitive function at year $4^{a}$ & $p$ value & Accelerated cognitive decline ${ }^{b}$ & $p$ value \\
\hline \multicolumn{5}{|l|}{ Entire sample $(\max N=831)$} \\
\hline Historical systolic BP & $1.00(0.98,1.01)$ & 0.635 & $1.01(0.99,1.03)$ & 0.228 \\
\hline $\begin{array}{l}\text { History of moderate/heavy vs never } \\
\text { smoked/history of light smoking }\end{array}$ & $2.01(1.38,2.92)$ & $<0.001$ & $1.64(1.14,2.34)$ & 0.007 \\
\hline Pack-years & $1.01(1.00,1.02)$ & 0.001 & $1.01(1.00,1.01)$ & 0.013 \\
\hline Historical $\mathrm{HbA}_{1 \mathrm{c}}$ & $1.24(1.02,1.49)$ & 0.029 & $1.21(1.00,1.45)$ & 0.046 \\
\hline \multicolumn{5}{|l|}{$\operatorname{Men}(\max N=430)$} \\
\hline Historical systolic BP & $1.00(0.97,1.02)$ & 0.651 & $1.02(1.00,1.05)$ & 0.053 \\
\hline $\begin{array}{l}\text { History of moderate/heavy vs never } \\
\text { smoked/history of light smoking }\end{array}$ & $2.09(1.25,3.52)$ & 0.005 & $1.61(0.97,2.66)$ & 0.065 \\
\hline Pack-years & $1.01(1.00,1.02)$ & 0.036 & $1.01(1.00,1.02)$ & 0.154 \\
\hline Historical $\mathrm{HbA}_{1 \mathrm{c}}$ & $1.34(1.02,1.77)$ & 0.035 & $0.95(0.73,1.24)$ & 0.706 \\
\hline \multicolumn{5}{|l|}{ Women $(\max N=401)$} \\
\hline Historical systolic BP & $1.00(0.98,1.02)$ & 0.932 & $1.00(0.98,1.02)$ & 0.802 \\
\hline $\begin{array}{l}\text { History of moderate/heavy vs never } \\
\text { smoked/history of light smoking }\end{array}$ & $1.83(1.06,3.17)$ & 0.030 & $1.66(0.99,2.77)$ & 0.054 \\
\hline Pack-years & $1.02(1.01,1.04)$ & 0.003 & $1.02(1.00,1.03)$ & 0.023 \\
\hline Historical $\mathrm{HbA}_{1 \mathrm{c}}$ & $1.14(0.88,1.45)$ & 0.326 & $1.50(1.15,1.96)$ & 0.003 \\
\hline
\end{tabular}

Data are ORs ( $95 \%$ CIs) comparing incremental odds of scoring in the lowest tertile of the respective distributions vs the highest tertile

Analyses are multiple logistic regression analyses that were performed separately for each risk factor. Never smoked/history of light smoking was defined as $<10$ pack-years. History of moderate/heavy smoking was defined as $\geq 10$ pack-years. Pack-years was untransformed in these analyses. Outcome variable is $g$ at year 4. Analyses for entire sample was adjusted for age, sex and in sex-stratified analyses for age. Cut-points for follow-up $g$ : lowest tertile $<-0.43$; medium tertile -0.43 to 0.48 ; highest tertile $>0.48$. Cut-points for follow-up $g$ adjusted for baseline $g$ : lowest tertile $<-0.39$; medium tertile -0.39 to 0.44 ; highest tertile $>0.44$

${ }^{a}$ Model of reduced cognitive function at year 4 was defined as scoring in the lowest tertile of $g$ at year 4

${ }^{\mathrm{b}}$ Model of accelerated 4 year cognitive decline was defined as scoring in the lowest tertile of year $4 g$ adjusted for baseline $g$

Historical $\mathbf{H b A}_{\mathbf{1 c}}$ Historical $\mathrm{HbA}_{1 \mathrm{c}}$ was unrelated to Mill Hill Vocabulary ( $p=0.311$ ), but was associated with lower $g$ and with accelerated decline in $g$ (Table 2). It was also significantly associated with decline on all tests except verbal memory (Logical Memory) and processing speed (Digit Symbol Coding; ESM Table 1). Adjustment for vascular disease, disease duration and Mill Hill Vocabulary (Table 2; ESM Table 1), or for glucose-lowering treatment (data not shown), did not alter the results. Each percentage $(11 \mathrm{mmol} / \mathrm{mol})$ increase in historical $\mathrm{HbA}_{1 \mathrm{c}}$ was associated with a $21 \%$ increased odds of accelerated 4 year decline when age and sex were controlled for (Table 3 ).

\section{Relative independence of vascular risk associations with cognition in total sample}

Historical $\mathrm{HbA}_{1 \mathrm{c}}$, historical systolic $\mathrm{BP}$, cholesterol and packyears were entered in a single linear regression model controlling for age and sex. Significant contributors to 4 year change in $g$ were pack-years $(-0.14 ; p<0.001)$, historical $\mathrm{HbA}_{1 \mathrm{c}}$ $(-0.10 ; p=0.006)$ and historical systolic BP $(-0.07 ; p=$ 0.052 ). The addition of vascular disease, duration of diabetes and Mill-Hill Vocabulary into the model did not alter the results: pack-years $(-0.11 ; p=0.004)$, historical $\mathrm{HbA}_{1 \mathrm{c}}$ $(-0.09 ; p=0.014)$ and historical systolic BP $(-0.07 ; p=$ $0.050)$ were each associated with a steeper 4 year decline in g. Cholesterol was not included in these models (both $p>0.10$ ).

\section{Vascular risk and cognition in stratified analyses}

Subgroup analysis by glucose-lowering treatment mode showed that findings on historical $\mathrm{HbA}_{1 \mathrm{c}}$ in the total sample were driven by insulin-treated patients: for this treatment group, effect sizes in linear regression analyses appeared to be particularly strong (fully adjusted model for 4 year decline in $g$, standardised $\beta-0.22 ; p<0.001)$. Findings on historical $\mathrm{HbA}_{1 \mathrm{c}}$ also appeared to be driven by female sex, whereas those for historical systolic BP were restricted to the subsample of men (ESM Table 2). When historical $\mathrm{HbA}_{1 \mathrm{c}}$, historical systolic BP, cholesterol and pack-years were entered in a single model controlling for age, vascular disease and duration of diabetes, significant predictors of a steeper 4 year decline were pack-years $(-0.13 ; p=0.011)$ and historical systolic BP $(-0.12 ; p=0.018)$ for the subsample of men ( $p>0.05$ for cholesterol and historical $\mathrm{HbA}_{1 \mathrm{c}}$, respectively), and pack-years 
$(-0.13 ; p=0.012)$ and historical $\mathrm{HbA}_{1 \mathrm{c}}(-0.16 ; p=0.003)$ for women ( $p>0.05$ for cholesterol and historical systolic BP, respectively). These sex-specific patterns of associations were supported by findings from logistic regression analyses (Table 3).

\section{Discussion}

Smoking history, long-term exposure to raised BP and poorer glycaemic control were independently associated with an accelerated late-life cognitive decline short of dementia. Overall effect sizes were modest but, compared with the findings on $\mathrm{BP}$, those for a measure of mean $\mathrm{HbA}_{1 \mathrm{c}}$ collected over a time period of up to 20 years prior to cognitive testing and for lifetime smoking history appeared to be more robust across the total sample. Independent of age and sex, a history of moderate to heavy smoking and each percentage increase in $\mathrm{HbA}_{1 \mathrm{c}}$ (an increase that is equivalent to $11 \mathrm{mmol} / \mathrm{mol}$ ) was associated with a $64 \%$ and $21 \%$ increased risk of an accelerated cognitive decline during 4 year follow-up, respectively. Cholesterol was associated only cross-sectionally with cognition. While our findings are not directly transferable to a clinical setting (since our definition of accelerated decline was arbitrary and sample-specific), they should serve to increase awareness of cognitive impairment and the potential importance of treatment of vascular risk factors in patients with type 2 diabetes.

To date, the relationships between cardiovascular risk factors and cognition have only infrequently been studied in people with diabetes. The present study has extended earlier evidence of cross-sectional associations of hypertension [24, 25] and poorer glycaemic control $[26,27]$ with reduced cognitive function in type 2 diabetes by demonstrating prospective associations with accelerated late-life cognitive decline. The association of smoking history with cognitive decline supports recent findings from the Fremantle Diabetes Study, in which midlife smoking was associated with the risk of cognitive impairment in later life [13]. Findings on glycaemic control in the present cohort are also consistent with a recent analysis of the Atherosclerosis Risk in Communities (ARIC) study, which reported that people with diabetes in midlife declined cognitively at faster rates during a 20 year follow-up period compared with diabetes-free individuals [28] and which additionally suggested a role for severity of hyperglycaemia [28]. In our study, the corresponding association of historical blood glucose levels with cognitive decline in people with diabetes in later life was particularly evident for tests of executive function, which again is consistent with findings from the ARIC study [28] and with reports of vulnerability of that domain in people with diabetes [29]. The suggestion of stronger findings for glycaemic control in insulin-treated patients in the present study may be due to the advanced disease stage and comorbidity in these patients and, in women, merits further investigation.

Many previous studies on a similar range of risk factors have investigated single factors, despite complex relationships among them. The present study makes an important advance by showing that associations of each of the risk factors identified as being predictive of cognitive decline were independent of one another. Although observational findings do not correspond to causality, it is possible that each factor is associated with cognition in separate pathophysiological pathways. Their modifiable nature suggests the potential for each risk factor as a separate target for intervention to reduce patients' risk of cognitive impairment. Observational data from studies of people with type 2 diabetes appear to support this possibility (e.g. with associations of antihypertensive treatment with reduced risk of dementia as an endpoint of cognitive decline [30]) but current evidence from trials is limited and inconclusive. In the Memory in Diabetes study of the Action to Control Cardiovascular Risk in Diabetes (ACCORD-MIND) trial, a trend was observed for decelerated cognitive decline in the treatment arm that was subjected to intensified glucose-lowering treatment [31]. However, some evidence suggests that hypoglycaemia (a side effect of more intensive therapy) may increase the rate of cognitive decline [32-35], thereby potentially counteracting the beneficial effects of improved glycaemic control. Future trials comparing glucose-lowering agents that cause different degrees of hypoglycaemia may help to clarify this. Trials on smoking cessation are complicated by confounding and are scarce even in the general population, with a single study showing decelerated 2 year cognitive decline in people who successfully quit smoking compared with those who were unsuccessful [36]. A Cochrane review of randomised trials in the general population concluded that antihypertensive therapy was of no benefit in slowing cognitive decline [37] and in the ACCORD-MIND study, intensive lowering of BP in people with type 2 diabetes had no effect on cognitive decline [38].

The present findings are consistent with those of a number of studies that have reported links of higher midlife or late-life cholesterol, or of lower HDL-cholesterol, with lower late-life cognitive function or with a steeper cognitive decline in people with diabetes $[11,39,40]$. However, these studies did not adjust for pre-morbid ability. Here, we found evidence for reverse causality: lower pre-morbid ability predisposed participants to a higher late-life level of cholesterol and to lower late-life cognitive function, and so confounding of previous cross-sectional (and potentially prospective) evidence is likely. In line with the present null findings for prospective associations of either cholesterol (without regard to lipid-lowering treatment) or of presence of hypercholesterolaemia (partly defined by use of lipid-lowering treatment), ACCORD-MIND revealed no effect of addition of fenofibrate to simvastatin therapy on 3 year cognitive decline [38]. 
The prospective design and extensive clinical and cognitive characterisation are clear strengths of the ET2DS. Adjustment for estimated peak pre-morbid ability helped to counteract potential reverse causality. The dropout of $22 \%$ over 4 years was relatively high but acceptable considering the diabetic status and age of the cohort. The associated survivor bias favouring healthier individuals to attend follow-up is likely to have led to an underestimate in effect sizes, so that associations may be stronger than reported here. Our overall results are further strengthened by the use of historical data. Despite data heterogeneity (historical data were available for between 1 and 65 readings), which may decrease the overall reliability of our findings, the approach substantially reduced the influence of measurement error compared with single measurement data. The calculation of a $g$ factor allowed the analysis of decline in overall cognitive function that was independent of the specific test battery used to measure cognitive function [41]. A resulting neglect of test-specific variance [42] was partly offset by additional consideration of findings from individual cognitive tests. Multiple testing will have increased the risk of type I statistical error and subgroup analysis by sex will have reduced statistical power, limiting the reliability of all findings and of those stratified by sex in particular. Replication in other cohorts with type 2 diabetes is therefore essential. Readers should also note that the category of accelerated cognitive decline was specific to the current, predominantly dementia-free cohort, and should not be mistaken for the presence of dementia. Finally, we did not consider the role of treatment of vascular risk, and so potential moderation of risk factor associations with cognitive decline by treatment cannot be excluded. The contributions of insulin or risk factor trajectories between baseline and year 4 follow-up to findings were also not assessed; such analyses are currently planned as part of future follow-up waves of the ET2DS.

Should associations of long-term exposure to vascular risk with cognitive decline prove to be causal, the present findings suggest that interventions targeting a range of vascular risk factors (rather than single factors such as hyperglycaemia only) may be needed to help reduce the risk of cognitive impairment in people who either have diabetes or who go on to develop type 2 diabetes in older age. Our findings support recent evidence that late-life cognitive change may be determined over the course of decades prior to the age at which deficits typically occur [28].

Overall, we have provided strong observational evidence showing that smoking history, higher BP and poorer glycaemic control are independently associated with accelerated late-life cognitive decline in people with type 2 diabetes. Further research into the usefulness of each risk factor as a target for intervention to reduce age-related cognitive decline in this patient population is now warranted.

Acknowledgements We thank all participants of the ET2DS.
Funding The sponsor for the ET2DS was the University of Edinburgh. The study was funded by the Medical Research Council (UK) and the Chief Scientist Office of the Scottish Executive. The funders had no other role in the design, analysis or writing of this manuscript. JFP, BMF and IJD are members of The University of Edinburgh Centre for Cognitive Ageing and Cognitive Epidemiology, part of the cross council Lifelong Health and Wellbeing Initiative (which has funding from the BBSRC, EPSRC, ESRC and MRC; G0700704/84698). None of the authors received payment from pharmaceutical companies or other agencies for the writing of this paper.

Access to research materials Samples and data of the ET2DS may be accessible subject to requests passing the study's usual data sharing and collaboration processes, which include protection of participant anonymity.

Duality of interest MWJS has received fees for speaking from Novo Nordisk, Eli Lilly and Pfizer and BMF has received honoraria for lecturing and attending advisory boards from Novo Nordisk, Eli Lilly, SanofiAventis, MSD, Janssen and Boehringer Ingelheim. All other authors declare that there is no duality of interest associated with their contribution to this manuscript.

Contribution statement JFP and MWJS conceived and designed the ET2DS and oversaw the acquisition and analysis of data. MK, JRM, CMR, SM and IF were involved in the collection of data. IF performed the literature search, the statistical analysis and drafted the manuscript with supervision from JFP, MWJS, BMF and IJD. MK, CMR, JRM, SM, IJD, BMF, MWJS and JFP were involved in the interpretation of findings, the drafting of the manuscript and its critical revision for important intellectual content. All approved the final version of the manuscript for publication. IF is the guarantor of this paper and as such had full access to all the data in the study, and takes responsibility for the integrity of the data and the accuracy of the data analysis.

Open Access This article is distributed under the terms of the Creative Commons Attribution 4.0 International License (http:// creativecommons.org/licenses/by/4.0/), which permits unrestricted use, distribution, and reproduction in any medium, provided you give appropriate credit to the original author(s) and the source, provide a link to the Creative Commons license, and indicate if changes were made.

\section{References}

1. Cukierman T, Gerstein HC, Williamson JD (2005) Cognitive decline and dementia in diabetes-systematic overview of prospective observational studies. Diabetologia 48:2460-2469

2. UnitedHealth Center for Health Reform \& Modernization (2010) The United States of Diabetes: challenges and opportunities in the decades ahead. United Health Group, Minnetonka

3. Takeda S, Sato N, Rakugi H, Morishita R (2011) Molecular mechanisms linking diabetes mellitus and Alzheimer disease: betaamyloid peptide, insulin signaling, and neuronal function. Mol Biosyst 7:1822-1827

4. Jackson K, Barisone GA, Diaz E, Jin L-W, DeCarli C, Despa F (2013) Amylin deposition in the brain: a second amyloid in Alzheimer's disease? Ann Neurol 74:517-526

5. Feinkohl I, Keller M, Robertson CM et al (2013) Clinical and subclinical macrovascular disease as predictors of cognitive decline in 
older patients with type 2 diabetes: the Edinburgh Type 2 Diabetes Study. Diabetes Care 36:2779-2786

6. Yaffe K, Blackwell T, Kanaya AM, Davidowitz N, Barrett-Connor E, Krueger K (2004) Diabetes, impaired fasting glucose, and development of cognitive impairment in older women. Neurology 63: 658-663

7. Okusaga O, Stewart MC, Butcher I, Deary IJ, Fowkes FGR, Price JF (2013) Smoking, hypercholesterolaemia and hypertension as risk factors for cognitive impairment in older adults. Age Ageing 42:306-311

8. Stewart MCW, Deary IJ, Fowkes FGR, Price JF (2006) Relationship between lifetime smoking, smoking status at older age and human cognitive function. Neuroepidemiology 26:83-92

9. Richards M, Jarvis MJ, Thompson N, Wadsworth MEJ (2003) Cigarette smoking and cognitive decline in midlife: evidence from a prospective birth cohort study. Am J Public Health 93:994-998

10. Anstey KJ, von Sanden C, Salim A, O'Kearney R (2007) Cigarette smoking and cognitive decline in midlife: evidence from a prospective birth cohort study. Am J Epidemiol 166:367-378

11. Stampfer MJ (2006) Cardiovascular disease and Alzheimer's disease: common links. J Intern Med 260:211-223

12. Umegaki H, Kawamura T, Umemura T, Kawano N (2015) Factors associated with cognitive decline in older adults with type 2 diabetes mellitus during a 6-year observation. Geriatr Gerontol Int 15: 302-310

13. Bruce DG, Davis WA, Starkstein SE, Davis TM (2014) Mid-life predictors of cognitive impairment and dementia in type 2 diabetes mellitus: The Fremantle Diabetes Study. J Alzheimers Dis 42(Suppl 3):S63-S70

14. Marioni RE, Strachan MWJ, Reynolds RM et al (2010) Association between raised inflammatory markers and cognitive decline in elderly people with type 2 diabetes The Edinburgh Type 2 Diabetes Study. Diabetes Care 59:710-713

15. Price JF, Reynolds RM, Mitchell RJ et al (2008) The Edinburgh Type 2 Diabetes Study: study protocol. BMC Endocr Disord 8:1827

16. Millan J, Pinto X, Munoz A et al (2009) Lipoprotein ratios: physiological significance and clinical usefulness in cardiovascular prevention. Vasc Health Risk Manag 5:757-765

17. Wechsler D (1997) Wechsler adult intelligence scale-3rd edition (WAIS-III). Pearson, San Antonio

18. Wechsler D (1987) Manual of the Wechsler memory scale-revised. The Psychological Corporation Limited, New York

19. Raven J, Raven JC, Court JH (1998) Manual for Raven's progressive matrices and vocabulary scales. Oxford Psychologists Press Limited, Oxford

20. Yuspeh RL, Vanderploeg RD (2000) Spot-the-word: a measure for estimating premorbid intellectual functioning. Arch Clin Neuropsychol 15:319-326

21. Deary IJ, Whalley LJ, Crawford JR (2004) An 'instantaneous' estimate of a lifetime's cognitive change. Intelligence 32:113-119

22. Spearman C (1904) 'General intelligence', objectively determined and measured. Am J Psychol 15:201-292

23. Willett JB (1997) Measuring change: what individual growth modeling buys you. In: Amsel E, Renninger KA (eds) Change and development: issues of theory, method, and application. Lawrence Erlbaum Associates Inc., Mahwah, pp 213-243

24. Elias PK, Elias MF, D'Agostino RB et al (1997) NIDDM and blood pressure as risk factors for poor cognitive performance. The Framingham Study. Diabetes Care 20:1388-1395
25. Lee YJ, Kang HM, Kim NK et al (2014) Factors associated for mild cognitive impairment in older Korean adults with type 2 diabetes mellitus. Diabetes Metab J 38:150-157

26. De Wet H, Levitt N, Tipping B (2007) Executive cognitive impairment detected by simple bedside testing is associated with poor glycaemic control in type 2 diabetes. S Afr Med J 97:1074-1076

27. Cukierman-Yaffe T, Gerstein HC, Williamson JD, Lazar RM, Lovato L, Miller ME et al (2009) Relationship between baseline glycemic control and cognitive function in individuals with type 2 diabetes and other cardiovascular risk factors: the action to control cardiovascular risk in diabetes-memory in diabetes (ACCORDMIND) trial. Diabetes Care 32:221-226

28. Rawlings AM, Sharrett AR, Schneider ALC et al (2014) Diabetes in midlife and cognitive change over 20 years: a cohort study. Ann Intern Med 161:785-793

29. McCrimmon RJ, Ryan CM, Frier BM (2012) Diabetes and cognitive dysfunction. Lancet 379:2291-2299

30. Johnson ML, Parikh N, Kunik ME et al (2012) Antihypertensive drug use and the risk of dementia in patients with diabetes mellitus. Alzheimers Dement 8:437-444

31. Launer LJ, Miller ME, Williamson JD et al (2011) Effects of intensive glucose lowering on brain structure and function in people with type 2 diabetes (ACCORD MIND): a randomised open-label substudy. Lancet Neurol 10:969-977

32. Feinkohl I, Aung PP, Keller M et al (2014) Severe hypoglycemia and cognitive decline in older people with type 2 diabetes: the Edinburgh Type 2 Diabetes Study. Diabetes Care 37:507-515

33. Whitmer RA, Karter AJ, Yaffe K, Quesenberry CPJ, Selby JV (2009) Hypoglycemic episodes and risk of dementia in older patients with type 2 diabetes. JAMA 301:1565-1572

34. Punthakee Z, Miller ME, Launer LE et al (2012) Poor cognitive function and risk of severe hypoglycemia in type 2 diabetes. Diabetes Care 35:787-793

35. Biessels GJ (2014) Hypoglycemia and cognitive decline in older people with type 2 diabetes: a bidirectional relationship. Diabet Hypoglycemia 6:11-14

36. Almeida OP, Garrido GJ, Alfonso H et al (2011) 24-month effect of smoking cessation on cognitive function and brain structure in later life. Neuroimage 55:1480-1489

37. McGuiness B, Craig D, Bullock R, Passmore P (2009) Statins for the prevention of dementia. Cochrane Database Syst Rev (2): CD003160. doi: 10.1002/14651858.CD003160.pub2

38. Williamson JD, Launer LJ, Bryan RN et al (2014) Cognitive function and brain structure in persons with type 2 diabetes mellitus after intensive lowering of blood pressure and lipid levels: a randomized clinical trial. JAMA Intern Med 174:324-333

39. Niu M-J, Yin F-Z, Liu L-X, Fang Y, Xuan X-M, Wu G-F (2013) Non-high-density lipoprotein cholesterol and other risk factors of mild cognitive impairment among Chinese type 2 diabetic patients. J Diabetes Complications 27:443-446

40. Reijmer YD, van den Berg E, Dekker JM et al (2012) Development of vascular risk factors over 15 years in relation to cognition: the Hoorn Study. J Am Geriatr Soc 60:1426-1433

41. Johnson W, Te Nijenhuis J, Bouchard TJ (2008) Still just 1 g: consistent results from five test batteries. Intelligence 36:81-95

42. Deary IJ, Penke L, Johnson W (2010) The neuroscience of human intelligence differences. Nat Rev Neurosci 11:201-211 